\title{
Measurement of amplified binary-modulated chirped laser pulses generated by different acousto-optic pulse shaping algorithms
}

\author{
Vladimir Ya. Molchanov, Konstantin B. Yushkov \\ National University of Science and Technology MISIS, \\ 4 Leninsky prospekt, Moscow 119049, Russia \\ Pavel V. Kostryukov \\ Lebedev Physical Institute of the Russian Academy of Sciences, \\ 53 Leninsky prospekt, Moscow 119991, Russia \\ Petr B. Gornostaev, Nikolay S. Vorobiev \\ Prokhorov General Physics Institute of the Russian Academy of Sciences, \\ 38 Vavilova str., Moscow 119991, Russia
}

\begin{abstract}
The paper belongs to the field of ultrafast optics. Acousto-optic pulse shaping has been studied experimentally for binary intensity modulation in a chirped pulse amplification laser system. Direct time-domain measurement of pulse front duration with a picosecond streak camera was performed for a Ti:sapphire regenerative amplifier. It was discovered that the sign of the second order dispersion produced by the acousto-optic dispersion delay line affects the modulation rise/fall time. Two algorithms for synthesis of ultrasonic waveforms for feeding the delay line, dispersive Fourier synthesis and the Gerchberg-Saxton algorithm, were compared. Minimum pulse front duration of $3.6 \mathrm{ps}$ for $3 \mathrm{~mJ}$ pulses with the linear chirp of $6.2 \mathrm{ps} / \mathrm{nm}$ at the wavelength of $795 \mathrm{~nm}$ was obtained.
\end{abstract}

Keywords: chirped pulse amplification, acousto-optics, regenerative amplifier, ultrashort pulse shaping

\section{Introduction}

Spectral shaping of phase-stretched broadband laser pulses is an effective way to obtain fast and programmable modulation in time domain. This technique uses a wellknown property of similarity between intensity profile of a chirped pulse and its power spectrum with second-order dispersion (SOD) as a scaling factor. Arbitrary modulated laser pulses are required in a number of applications including communications, ultrafast laser material processing, and inertial confinement fusion. Frequencyto-time mapping is a robust and cost-effective method for generating arbitrary $\mathrm{RF}$ waveforms in $\mathrm{GHz}$ and $\mathrm{THz}$ bands $[1+3$. Chirped laser pulses and pulse trains can improve efficiency of material ablation and nanoparticle generation via tailoring specific thermodynamic states of irradiated matter 4 -7]. It is also argued that using spreadspectrum pulses instead of amplitude-modulated quasimonochromatic ones can sufficiently suppress laser plasma instabilities and induced Brillouin scattering in fast ignition fusion schemes [8 11].

Email address: konstantin.yushkov@misis.ru (Konstantin B. Yushkov)
Chirped pulse amplification (CPA) is a versatile and highly scalable method for obtaining high-energy broadband laser pulses [12 14]. The first amplification stage in CPA systems is usually a regenerative amplifier (RA). A straightforward way to maximize and stabilize the energy of modulated pulses is placing a pulse shaper before a saturated RA. This cancels strict requirements of laser damage threshold of the pulse shaper and allows compensating for insertion losses in the shaper by the amplifier gain. The price for this is possible distortion of the pulse envelope in a saturated amplifier. Though the pulse distortions can be reduced by adaptive feedback and iterative algorithms [15, 16, this path is often resource and time demanding. Adaptive pre-shaping concept in CPA was experimentally assessed by Ohno et al. demonstrating adaptive pulse compression, compensation for gain narrowing, and pulse train generation using an acousto-optic (AO) filter with digital feedback [15. However, the algorithm for RF signal optimization was running over the search space of 32 variables per $100 \mathrm{~nm}$ spectral bandwidth, therefore spectral resolution was artificially limited at least by an order of magnitude. It also required a few thousand iterations including FROG phase retrieval so this technique was far from real-time operation. 


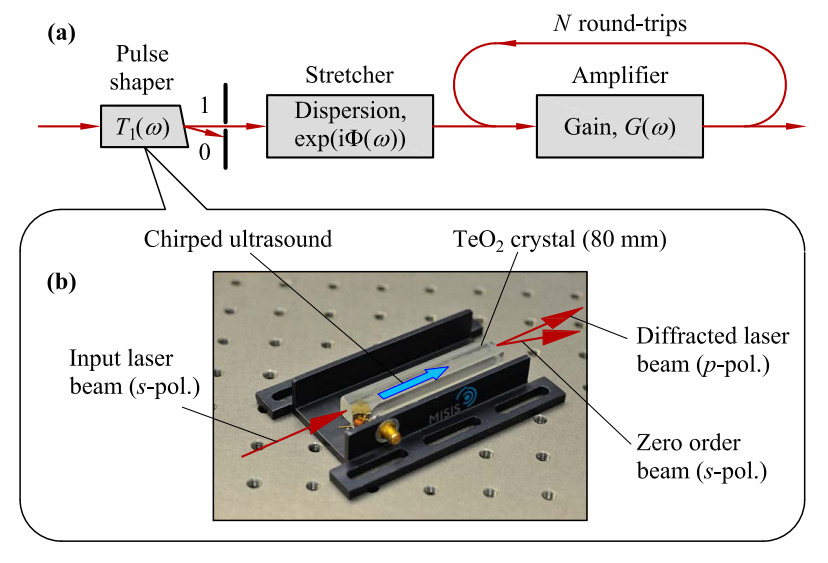

Figure 1: Apparatus for spectral preshaping in CPA: (a) system architecture; (b) paratellurite high-resolution AODDL.

In this work, we report direct time-domain assessment of a programmable millijoule-level pulse source based on a high-resolution pulse shaper and a Ti:sapphire RA. Laser pulse envelopes were synthesized at the stretcher input with an AO dispersion delay line (AODDL) [17] and measured at the RA output with a picosecond-resolution streak camera 18, 19. It was found that the rise/fall time of chirped binary modulated laser pulses is affected by the sign of SOD introduced by acousto-optic diffraction in the AODDL. Two different algorithms for synthesis of RF waveforms, dispersive Fourier synthesis (DFS) and the Gerchberg-Saxton (GS) algorithm, were compared. The causes of variable pulse front duration are investigated. The developed instrumentation and measurement technique can be considered as a tool for characterization CPA systems in applications requiring complex time-domain optical waveform synthesis [20].

\section{Experimental apparatus and techniques}

\subsection{Spectral pre-shaping}

The AODDL for pulse shaping was placed at the input of the CPA system, i.e. before the stretcher as shown in Fig. 1. The output beam of the AODDL is the first diffraction order that enables phase and amplitude pulse shaping, i.e. the transmission $T_{1}(\omega)$ is a complex-valued function and has a variable absolute value $\left|T_{1}(\omega)\right|$. In this system architecture, high accuracy and modulation contrast is required, because the pulses are amplified after the shaper, and no feedback loop is used. The benefits of this approach are fast and deterministic synthesis of chirped laser pulse envelopes, while the challenge is to maintain the pulse shape during amplification because of gain narrowing and saturation effects in the RA. As we will show in Results section, the output performance depends on the algorithm for synthesis of the RF waveforms applied to the AODDL.

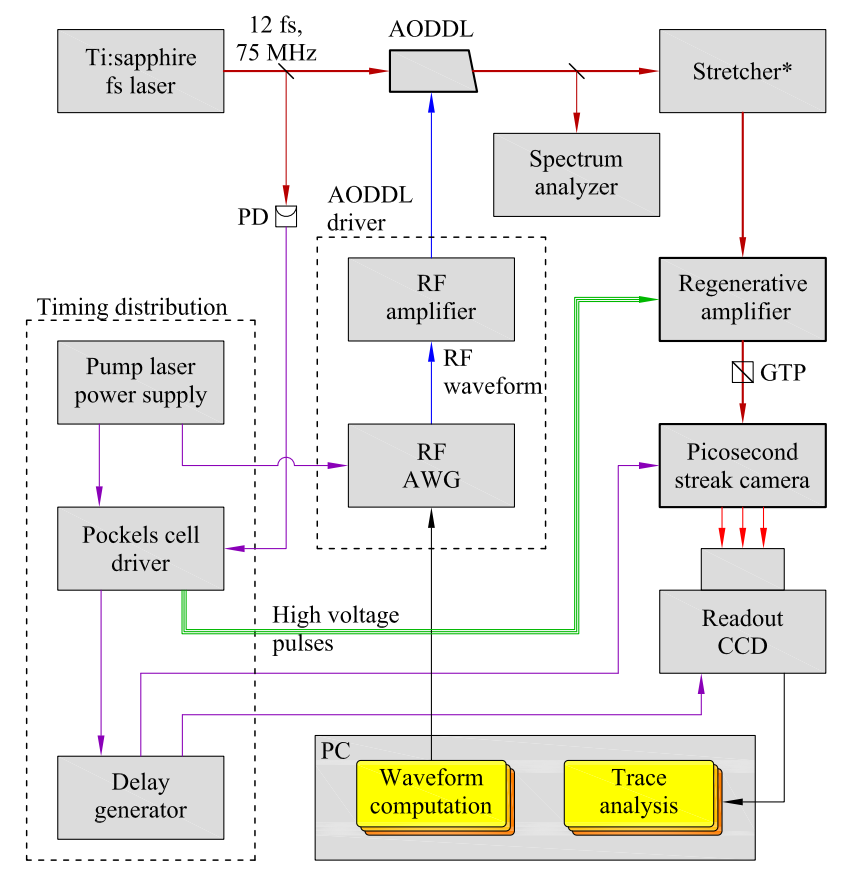

Figure 2: Experimental setup for time-domain characterization of the programmable CPA source. The AODDL performs spectral shaping of pulses seeded to the Ti:sapphire RA; chirped laser pulses are measured by the streak camera after the amplifier without recompression.

\subsection{Experimental setup}

The experimental setup is schematically shown in Fig. 2 The laser source was the 12-fs Ti:sapphire master oscillator Femtosource Synergy (Femtolasers, Austria). Laser radiation bandwidth was $80 \mathrm{~nm}$ FWHM and $130 \mathrm{~nm}$ FWTM centered at $795 \mathrm{~nm}$. The beam was spectrally shaped by the high-resolution AODDL designed and fabricated inhouse (NUST MISIS, Russia). The driver for the AODDL consisted of the arbitrary waveform generator (AWG) N8241A (Agilent, USA) and $25 \mathrm{~W}$ broadband RF amplifier 25A250A (Amplifier Research, USA). The diffracted beam was chirped by the Martinez stretcher and was used to seed the RA with $3 \mathrm{~mJ}$ output pulse energy REUS (Avesta Project Ltd., Russia). Additionally, the diffracted spectrum was measured with the optical spectrum analyzer 86142B (Agilent, USA). At the amplifier output, the beam was attenuated by a pair of Glan-Taylor polarizers (GTP) GT10-A (Thorlabs, USA) and variable neutral density filters and focused at the photocathode of the streak camera PS-1/S1 (General Physics Institute, Russia). Data readout from the streak camera was performed with a CCD camera Alta-U32 (Apogee Imaging Systems Inc., USA).

Synchronization and timing started from the pump laser power supply LPS-2226 (Lotis Tii, Belarus) and from the femtosecond pulse train at the master oscillator output. The Pockels cell driver of the RA controlled the number of resonator round-trips in the amplifier and distributed trigger signals for the streak camera and for the readout. Additionally, a delay generator DG535 (Stanford Research 
Systems, USA) was used for precise timing of the streak camera.

The AWG and the CCD were controlled by the personal computer (PC). The RF waveforms were programmed with a custom MATLAB script using fast Fourier transform (FFT) for waveform synthesis 21. The waveforms were stored in the AWG volatile memory and initiated with the external trigger. Synchronization of the RF waveform with the seed pulse injection into the RA resonator was controlled by the software to keep maximum diffraction efficiency of the pulse bunch at the AODDL output when the pulse picker opens.

The stretcher in standard configuration has the SOD of $\sim 2 \cdot 10^{6} \mathrm{fs}^{2}$ providing the chirp of $\sim 6 \mathrm{ps} / \mathrm{nm}$ at the output in $100 \mathrm{~nm}$ bandwidth. The optical setup could be reconfigured to add the second pass through the stretcher and thus to double pulse chirping in the RA. The stretcher could be also replaced by the Treacy grating compressor providing the same amount of SOD with negative sign.

\section{3. $A O D D L$}

In the experiments, we used a high-resolution AODDL with the interaction length $L=80 \mathrm{~mm}$ in paratellurite and the beam aperture $a=4 \mathrm{~mm} 22$. The time aperture was equal to $T_{\mathrm{a}}=102.4 \mu \mathrm{s}$. The sampling-limited resolution for the $100 \mathrm{~nm}$ spectral bandwidth equals to $0.05 \mathrm{~nm}$ (2346 samples according to Nyquist criterion). To assure high accuracy of spectral modulation over the whole bandwidth, we retained RF waveform sidelobes in time domain [21, 23. In this case, the maximum effective duration of a linearly chirped $\mathrm{RF}$ waveform was $T_{0}=$ $0.5 T_{\mathrm{a}}=51.2 \mu \mathrm{s}$, and the range of programmable SOD was respectively a half of available for this crystal length (maximum SOD for this crystal configuration is $38000 \mathrm{fs}^{2}$ in $100 \mathrm{~nm}$ bandwidth). The sign of the RF waveform chirp in the AODDL could be changed by the software. The sign of the chirp corresponds to the sign of the SOD produced by the AODDL. This SOD adds up to passive dispersion of the paratellurite crystal $\left(b_{2} \approx 4.0 \cdot 10^{4} \mathrm{fs}^{2}\right.$ for ordinary wave and $4.7 \cdot 10^{4} \mathrm{fs}^{2}$ for extraordinary wave). The other experimentally relevant parameters of the AODDL are the phase velocity of ultrasound $v=641 \mathrm{~m} / \mathrm{s}$, the acoustic walk-off angle $\psi=40.6^{\circ}$, and the birefringence in the interaction direction $\Delta n=0.094$.

Fig. 3 shows an example of spectral and time domain characterization of the modulated optical pulse at the amplifier input. The full transmission window of the AODDL is set to $150 \mathrm{~nm}$. The spectrum is modulated with a periodic pattern of stop bands, $2.4 \mathrm{~nm}$ each, and a dedicated narrow notch mark at the spectrum center. A combination of binary modulation by the AODDL and phase stretching of the pulses results in multi-GHz intensity modulation that can be directly measured with a picosecond streak camera 3 . One stop band at $\sim 786 \mathrm{~nm}$ is intentionally skipped in the modulation pattern to clearly distinguish between short-wavelength and long-wavelength part of the spectrum at the streak camera trace. This trace was used
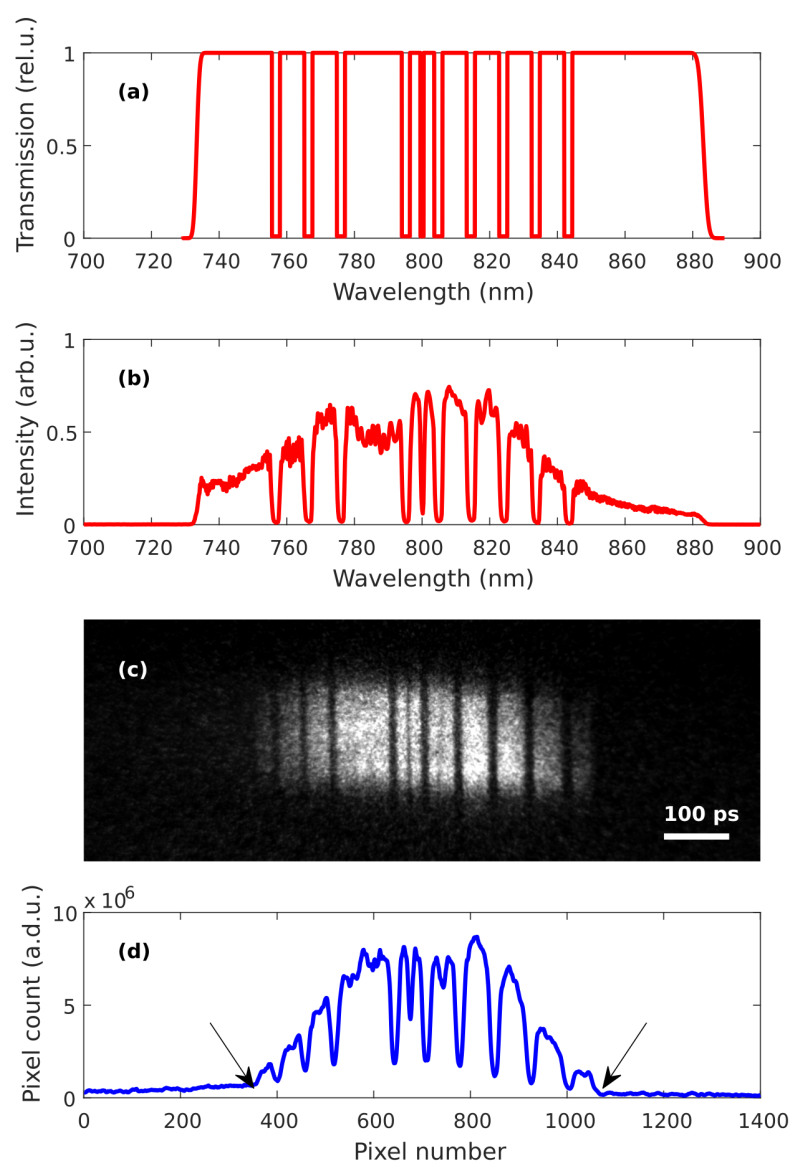

Figure 3: Test optical waveform generated by the AODDL: (a) userdefined spectrum with binary modulation; (b) measured optical spectrum; (c) recorded chirped pulse trace; (d) time-domain intensity profile. Arrows in the chirped pulse trace correspond to the stretcher cut-off wavelengths 750 and $850 \mathrm{~nm}$.

to find the actual value of the pulse chirp, which was 6.2 $\mathrm{ps} / \mathrm{nm}$. A simplified modulation pattern with a single stop band at the center of the spectrum was used in further experiments.

\subsection{RF waveform synthesis}

DFS is a technique based on FFT for synthesis of RF waveforms with given duration, bandwidth, and transmission magnitude by means of applying a quadratic spectral phase [21, 24]. This algorithm demonstrated high accuracy in synthesis of arbitrary complex spectral transmission functions $\widetilde{S}(\omega)$ including phase-only pulse shaping algorithms [25, 26]. Numerical procedure for DFS is similar to one-dimentional Fresnel transform [27]. The complexvalued $\mathrm{RF}$ waveform is calculated as

$$
\begin{aligned}
& S_{0}(t) \exp \left[i \Psi_{2}\left(t-t_{0}\right)^{2}+i \Phi_{0}(t)\right]= \\
& \operatorname{FT}\left\{\widetilde{S}(\Omega) \exp \left[i B_{2}\left(\Omega-\Omega_{0}\right)^{2}\right]\right\}
\end{aligned}
$$

where FT is the Fourier transform operator, $B_{2}$ is the acoustic SOD coefficient, $\Psi_{2}$ is the acoustic quadratic phase 
coefficient, and $\Phi_{0}(t)$ is non-quadratic phase of the acoustic signal. Bijection between optical frequencies $\omega$ and ultrasonic frequencies $\Omega$ is originating from phase matching and can be written as

$$
\Omega=\frac{v}{c} \Delta n(\omega) \omega
$$

Quadratic phase coefficients in Eq. (1) are determined only by the waveform duration $T_{0}$ and the bandwidth $\Delta \Omega$ :

$$
\Psi_{2}= \pm \frac{\Delta \Omega}{T_{0}}
$$

and

$$
B_{2}= \pm \frac{T_{0}}{2 \Delta \Omega}
$$

The amount of optical SOD $b_{2}$ is proportional to $B_{2}$ in linear approximation (i.e. neglecting dispersion of $\Delta n$ ):

$$
b_{2}=\frac{2}{\cos \psi}\left(\frac{v}{c} \Delta n\right)^{2} B_{2} .
$$

Plus sign in (3) and (4) corresponds to positive SOD produced by the Bragg grating, and minus sign corresponds to the same amount of negative SOD. Non-quadratic phase term $\Phi_{0}(t)$ in l.h.s. of Eq. (1) describes deviations of instantaneous frequency from strictly linear sweep.

Undesired phase-amplitude couplings in DFS method arise from a finite bandwidth of the shaper. The effect is strong when the spectral amplitude significantly varies within the passband of the filter. To study the effect of phase-amplitude couplings we compared DFS with a different waveform synthesis algorithm providing strictly linear frequency sweep. We applied the GS algorithm [16], which minimized phase retrieval error using iterative FFT, to eliminate nonlinearity of the frequency sweep and to retain binary modulation of the spectral amplitude $\left|\widetilde{T}_{1}(\omega)\right|$.

The GS algorithm was implemented as follows. We consider the spectral magnitude $\widetilde{S}(\Omega)$ as given and iteratively search for the additional spectral phase term $\phi(\Omega)$ to obtain in quadratic-only temporal phase after the Fourier transform:

$$
\begin{aligned}
& S(t) \exp \left[i \Psi_{2}\left(t-t_{0}\right)^{2}\right]= \\
& \operatorname{FT}\left\{\widetilde{S}(\Omega) \exp \left[i B_{2}\left(\Omega-\Omega_{0}\right)^{2}+i \phi(\Omega)\right]\right\}
\end{aligned}
$$

where $S(t)$ and $\phi(\Omega)$ are unknown. The coefficients $\Psi_{2}$ and $B_{2}$ are the same as in Eq. (1). Starting from $\phi_{0}(\Omega)=0$, we find the waveform amplitude,

$$
S_{n}(t)=\left|\operatorname{FT}\left\{\widetilde{S}(\Omega) \exp \left[i B_{2}\left(\Omega-\Omega_{0}\right)^{2}+i \phi_{n}(\Omega)\right]\right\}\right|,
$$

and the spectral phase at the next iteration is

$$
\begin{array}{r}
\phi_{n+1}(\Omega)=\arg \mathrm{FT}^{-1}\left\{S_{n}(t) \exp \left[i \Psi_{2}\left(t-t_{0}\right)^{2}\right]\right\}- \\
B_{2}\left(\Omega-\Omega_{0}\right)^{2} .
\end{array}
$$
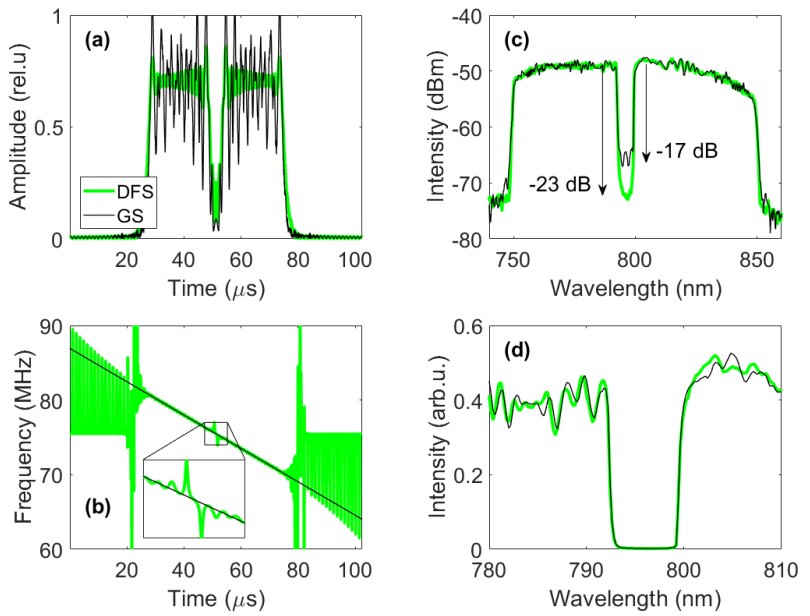

Figure 4: RF waveform and corresponding spectrum of diffracted light obtained with DFS and GS algorithms: (a) RF signal amplitude; (b) RF signal instantaneous frequency; (c) diffracted optical spectrum, logarithmic scale; (d) diffracted optical spectrum, zoomin in linear scale.

Finally, when the GS algorithm converges to a stationary solution, we substitute $\phi_{N}(\Omega)$ into (7) to calculate $S_{N}(t)$ and define the $\mathrm{RF}$ waveform as

$$
U(t)=\operatorname{Re}\left\{S_{N}(t) \exp \left[i \Psi_{2}\left(t-t_{0}\right)^{2}\right]\right\}
$$

The results of applying DFS and GS algorithms for generating spectral notch filtering with sharp modulation edges are shown in Fig. 4. Instantaneous frequency is calculated as the derivative the waveform phase. Those signals correspond to the width of the stop band of $7.2 \mathrm{~nm}$ at the central wavelength of $795 \mathrm{~nm}$. The plots show the amplitude and the instantaneous frequency of RF signals and measured spectra of diffracted light of initial DFS signals, and after 60 iterations of the GS algorithm. The GS algorithm enhances the amplitude ripples in the $\mathrm{RF}$ waveform compared to DFS and provides good spectral contrast and the same steepness of binary spectral modulation.

\subsection{Experimental parameters}

In the experiments, we applied notch spectral modulation with the stop band of $7.2 \mathrm{~nm}$ centered at $795 \mathrm{~nm}$. This stop band is 30 times wider than the AODDL passband and the corresponding dip in the chirped pulse envelope is $\sim 10$ times wider than the resolution of the streak camera.

The CPA system was used in three different configurations. The pulses were chirped by: 1) single-pass stretcher (standard), 2) double-pass stretcher (twofold pulse stretching), and 3) compressor (negative chirp). The stretching factors in these configurations are further referred to as 1 , 2 , and -1 respectively. Using the compressor instead of the stretcher was aimed at distinguishing between the effect of shorter and longer wavelengths in the system. Full number of the pulse roundtrips in the z-shaped RA resonator was 18 , and the output pulse energy is $3 \mathrm{~mJ}$ at the 

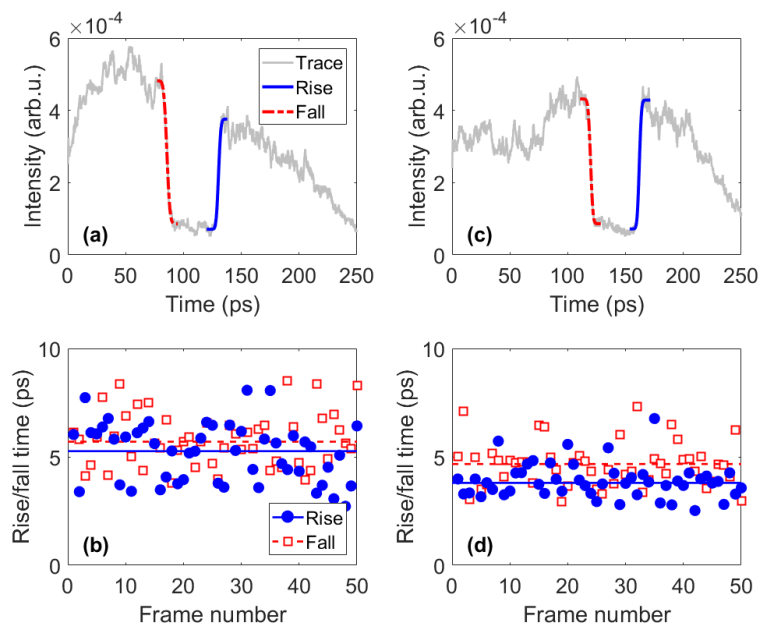

Figure 5: Experimental data analysis: $(\mathrm{a}, \mathrm{c})$ a streak camera trace and slope fitting; (b,d) front durations in a series of frames (dots) and median values for each series (lines). The traces were obtained for different SOD signs: (a,b) $B_{2}>0$; $(\mathrm{c}, \mathrm{d}) B_{2}<0$.

resonator output. We also performed the measurement at half passes through the RA (9 round-trips), i.e. the case when the RA is far from saturation.

For each setting of the CPA system, we measured the traces both at positive and negative SOD of the RF waveform $B_{2}\left(\right.$ and $\left.\Psi_{2}\right)$.

\subsection{Trace acquisition and processing}

The streak camera traces were captured with a CCD. The scale of the images was $5.6 \mathrm{pix} / \mathrm{ps}$, and the minimum resolvable element of the streak camera was 3.5 ps that corresponded to CCD 20 pixels eliminating discretization artifacts.

Each trace was converted to one-dimensional array by averaging over 150 pixels in transverse direction. Then the slopes were approximated as

$$
I(t)=A \operatorname{erf} \frac{t-B}{C}+D
$$

with four independent coefficients $A, B, C$, and $D$ using nonlinear least square fit in MATLAB. The rise/fall time at $10-90 \%$ levels was calculated as $\tau_{r}=1.81 C$. Descriptive statistics were used to analyze the data. The sample distribution median was used as an estimate of average rise/fall time and 25-75\% quantiles were used as the estimate of dispersion in each series of measurements.

\section{Results}

Binary spectral amplitude modulation is a straightforward way to obtain steep envelope modulation. Softwaredefined spectral amplitude step between 0 to 1 had the width of $0.05 \mathrm{~nm}$ (one sampling interval in the frequency domain) that ensured that it can be considered as a binary unit step. The measurements of at $10-90 \%$ rise/fall time
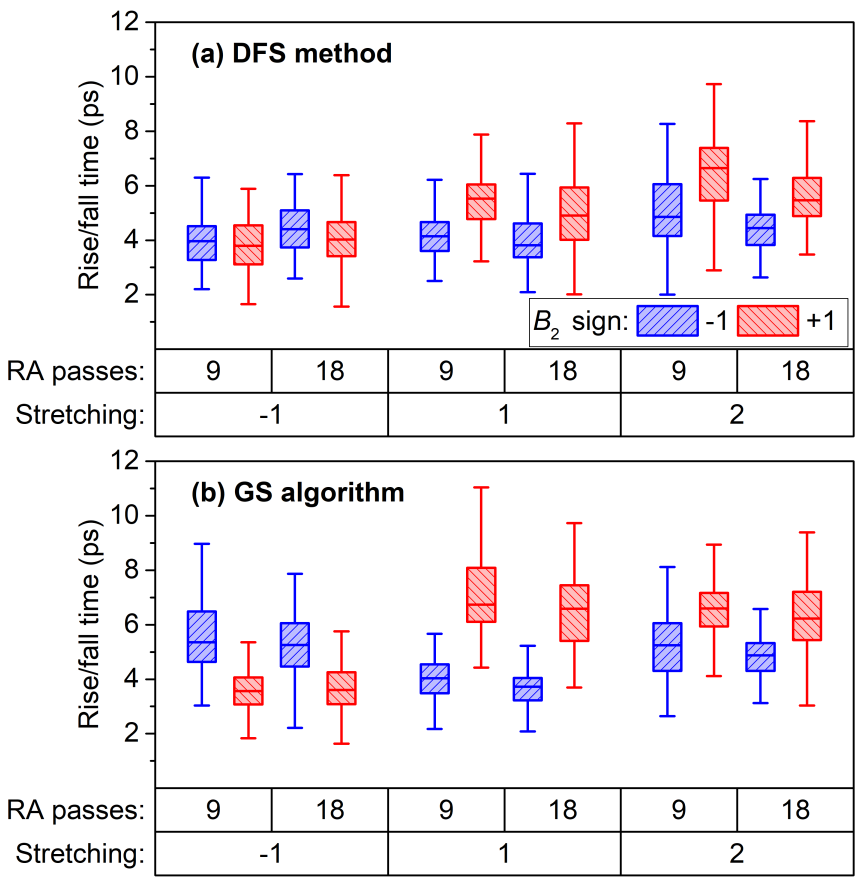

Figure 6: Statistics of rise/fall time at different experimental conditions: (a) RF waveforms generated by DFS method; (b) RF waveforms generated by GS algorithm method. The box plot shows the median and $25 \%-75 \%$ quantiles; the whiskers are at 1.5 times interquartile range.

ranged from $3.6 \pm 1.0$ to $6.7 \pm 2.0$ ps depending on experimental settings. Corresponding front width in spectral domain was $0.57 \mathrm{~nm}$ as shown in Fig. 4 (d).

Experimental trace at the standard CPA system configuration (stretching factor $+1,18$ round trips in the resonator) is shown in Fig. 5 for different $B_{2}$ signs: (a) and (b) for $B_{2}>0$; (c) and (d) for $B_{2}<0$. In each series, 50 individual traces were analyzed to find median values of rise/fall time.

Total number of analyzed traces after rejection of outliers was 1304, grouped in 24 experimental series with the minimum of 31 traces per series. The rise time and the fall time corresponding to two edges of the stop band were calculated for each trace. Analysis of the dataset did not reveal any statistically significant difference between the rise time and the fall time.

The summary of statistics is plotted in Fig. 6. The plot shows that there is no effect of the number of resonator round-trips on the rise/fall time. However, there is the difference between positive and negative $B_{2}$ values. This difference is expressed much stronger for the GS algorithm than for the DFS. The GS algorithm also provides the evidence that the effect is inverted when the compressor is used instead of the stretcher (the stretching factor of -1 in Fig. 6(b)). 


\section{Discussion}

\subsection{Hypotheses overview}

In the experiments, we observed that the modulation rise/fall time depends on the sign of AO-induced SOD (5). This effect was reproducible under different experimental conditions. Besides the parameters described in Fig. 6 (number of passes in the RA and the stretching factor), we investigated the effects of the AODDL misalignment, geometry of the seed beam coupling with the resonator mode, absolute value of the SOD, and horizontal flipping of the seed beam. In all cases this result retained, except extreme misalignment of the AODDL or the beam injected into the resonator.

There are several effects that may cause the dependence of the modulation rise/fall time on the SOD sign. Since the rise and fall time in each measurement are the same within the measurement error, small asymmetry of the AODDL transmission function can not be the reason for this effect. Another factor that can contribute to the front duration is temporal dispersion.

The first possible reason of temporal dispersion is spatial dispersion. The angular chirp causes temporal dispersion because of the optical path difference 28]. For the same reason, spatial chirp may also cause a group delay in an anisoplanatic (non-spatially-invariant) optical system. Unlike a parallel-grating Treacy compressor, antiparallelgrating stretchers are principally anisoplanatic. Spatial chirp and divergence of the beam at the stretcher input may cause angular dispersion and pulse front tilt, i.e. the group delay dependent on the lateral beam displacement 29 , 30 .

The second source of temporal dispersion is the influence of AO phase modulation. Diffraction of ultrashort pulses by chirped ultrasound creates quadratic SOD according to Eq. (5), however an additional group delay can appear because of 1) phase-amplitude couplings or 2) imperfectness of the grating itself. In the first case, residual non-quadratic spectral phase is a principal effect of spectral amplitude modulation by a finite-resolution pulse shaper. In the second case, it is a property of the grating synthesis algorithm, which, in general, can add variations of the group delay from a linear function. As it will be shown in Sec. 4.3. only the first factor exists in the DFS waveform synthesis method, while the second factor dominates for the GS algorithm with linearized frequency modulation.

The following discussion gives quantitative estimates of those effects in the particular experimental setup.

\subsection{Spatial and angular chirp}

Previously, McCabe et al. found that the only one type of spatio-temporal coupling in quasicollinear AO devices is lateral shift of the output beam proportional to the group delay 31. Osvay et al. found that angular deviation and angular dispersion are proportional to the absolute value of SOD and attributed this effect to thermal gradients at high mean RF power 32. Indeed, even at a moderate average $\mathrm{RF}$ power of $\sim 1 \mathrm{~W}$, the stationary temperature gradient in a paratellurite crystal can be as high as $1 \mathrm{~K} / \mathrm{mm}$ along the optical beam path 33 . In our experiments, the AODDL was running in a triggered mode with the duty cycle of $1: 1000$, so the average power dissipated in the $\mathrm{TeO}_{2}$ crystal was at a negligible level of few milliwatt.

Anikin et al. showed that there are two types of angular dispersion in AO filters 34. The first type of angular chirp is caused by dispersion of birefringence and manifests itself as beam scanning under phase matching condition. This type of the angular chirp can be efficiently compensated by the output wedge of the crystal. The second type of angular chirp is caused by finite diffraction bandwidth. Simultaneous compensation of both types of the angular dispersion with the same wedge configuration is impossible.

The AODDL used in this work was designed for ordinary (i.e. fast) input light beam with the output wedge of the crystal compensating for the angular scanning of the diffracted extraordinary wave. Calculation of the residual angular chirp of both types gives the value $1.1 \times 10^{-2}$ $\mathrm{mrad} / \mathrm{nm}$ at $\lambda=800 \mathrm{~nm}$. Besides that, the optical beam is diffracted by the acoustic beam with a finite aperture $a$, resulting in FWHM angular divergence of the output beam

$$
\Delta \theta \approx 0.9 \lambda / a=0.18 \mathrm{mrad}
$$

This beam divergence adds up to the angular chirp.

Geometrical interpretation of the spatial chirp at the AODDL output is shown in Fig. 7. The RF waveform with effective duration $T_{0}<T_{a}$ fills a part of the crystal. The wave vector diagram in Fig. 7 (b) shows the directions of the diffracted beam wave vector and Poynting vector. Inversion of the sign of $B_{2}$, Fig. 7 (c) and (d), changes the position where different wavelengths emerge from the interaction area, but does not affect the dispersion of the diffraction angle. Thus, the sign of $B_{2}$ changes only the spatial chirp of the diffracted beam, but does not change its angular chirp.

Two factors that determine the spatial chirp at the AODDL output are the dispersion of the diffracted ray direction angle $\gamma$, and the spatial extension of the chirped ultrasonic Bragg grating along the optical beam path. Only the second of those two factors depends on the sign of RF chirp, therefore total spatial chirp of the diffracted beam changes with the sign of $B_{2}$. The value of $\gamma$ can be found from the wave vector diagram, Fig. 7 (b). For numerical estimates, it is enough to represent $\gamma(\lambda)$ through the Taylor series expansion:

$$
\gamma(\lambda)=\gamma_{0}+\gamma_{1}\left(\lambda-\lambda_{0}\right)
$$

The series coefficients $\gamma_{0}$ and $\gamma_{1}$ are determined by the direction of quasicollinear diffraction in $\mathrm{TeO}_{2}$ crystal. In our case, the diffraction geometry is $44.4^{\circ}$ to the optic axis [001] of the $\mathrm{TeO}_{2}$ crystal. Optical walk-off angle for the diffracted extraordinary wave at $\lambda=800 \mathrm{~nm}$ is $\beta=3.63^{\circ}$, 
(a)

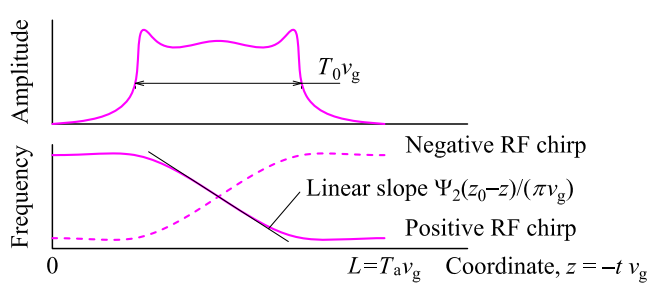

(b)

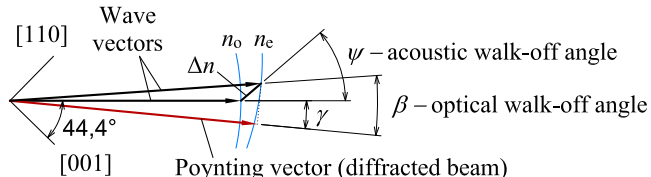

[001] Poynting vector (diffracted beam)

(c)

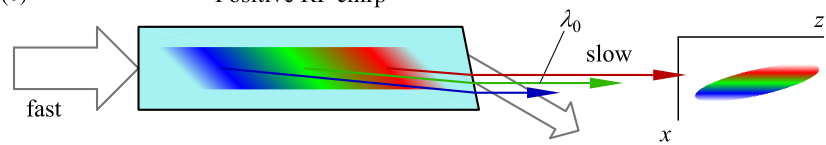

(d)

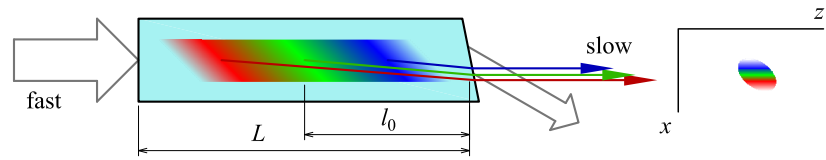

Figure 7: Spatial chirp of diffracted pulses at the AODDL output: (a) RF waveform; (b) wave vector diagram of phase matching; (c) ray path with positive RF chirp; (d) ray path with negative RF chirp.

which is close to the maximum. The coefficients in Eq. 12 are $\gamma_{0}=37 \mathrm{mrad}$ and $\gamma_{1}=-0.04 \mathrm{mrad} / \mathrm{nm}$.

The dependence of the phase-matching position $l$ in the crystal is determined by the derivative

$$
\frac{d l}{d \lambda}=-\frac{4 \pi B_{2} v^{2} \Delta n(\lambda)}{\lambda^{2} \cos \psi}
$$

and position $l_{0}$ of the ultrasonic waveform in the crystal (see Fig. 7, d). Obviously, $l_{0}$ depends on the delay between start of the RF waveform and triggering of the pulse picker that injects the pulse into the RA resonator. From 12 and 13 it follows that the spatial chirp can be expressed as

$$
\frac{d x}{d \lambda}=-\frac{4 \pi B_{2} v^{2} \Delta n(\lambda)}{\lambda^{2} \cos \psi} \tan \gamma_{0}+l_{0} \gamma_{1} .
$$

The quadratic phase (3) corresponding to maximum stretching of the ultrasonic waveform in the crystal for the experimental settings was $\Psi_{2}=1.4 \mu \mathrm{s}^{-2}$, and $B_{2}=0.178 \mu \mathrm{s}^{2}$ (corresponding optical SOD $(5)$ is $b_{2} \approx 19000 \mathrm{fs}^{2}$ ). The first summand in Eq. (14) equals $\pm 5.0 \cdot 10^{-3} \mathrm{~mm} / \mathrm{nm}$, while the second summand is only $-0.16 \cdot 10^{-3} \mathrm{~mm} / \mathrm{nm}$. We see that even large crystal length $L=80 \mathrm{~mm}$ does not cause a sufficient effect of $\gamma_{1}$ on the spatial chirp. Thus, we can state that changing the sign of SOD also changes the sign of the spatial chirp without significant change of the absolute value of $0.5 \mathrm{~mm}$ for the $100 \mathrm{~nm}$ bandwidth.

After the AODDL, the beam passes through the Martinez stretcher, of which proper alignment ensures the linear angular chirp below $10^{-3} \mathrm{mrad} / \mathrm{nm}$ leaving the intrin- sic cubic angular chirp of $4 \cdot 10^{-7} \mathrm{mrad} / \mathrm{nm}^{3}$. The measurements of modulation rise/fall time were made at the wavelength offset of $\pm 3.6 \mathrm{~nm}$ from the central wavelength, therefore the angular chirp of the stretcher is negligible compared to the chirp at the AODDL output. Finally, the distance from the AODDL to the first strike of the stretcher grating input was $2.7 \mathrm{~m}$, therefore the spatial chirp was approximately $20 \%$ of lateral wavelength displacement originating from the angular chirp at this distance.

\subsection{Group delay induced by AO modulation}

Apart from spatial and angular chirps, the steepness of intensity modulation can be affected by the group delay caused by the non-quadratic spectral phase of the RF waveform, which imprints on spectral phase of diffracted light, or by phase-amplitude couplings in the AO Bragg grating. This hypothesis was tested by comparison of two different algorithms for grating synthesis. The DFS method provides pure quadratic spectral phase, while the GS algorithm was used to generate the waveform with linear frequency modulation. The difference between the modulation rise/fall time obtained by DFS and GS algorithms gives the evidence that the pulse fronts are affected by non-quadratic terms of the spectral phase. Those terms correspond to local variations of the group delay.

For estimation of the group delay, we calculated the spectrum of the RF waveform $U(t)$, scaled it from acoustic to optical frequencies using Eq. (2), and convolved it with the AODDL transmission function. Then the group delay was calculated as the derivative of the spectral phase. Within this model, the change of the SOD sign changes only the sign but not the absolute value of the group delay. Calculated transmission spectra and the group delay are shown in Fig. 8 (a) and (b). Initial RF waveforms were taken from Fig. 4 . Despite pure quadratic phase in the initial spectrum of the DFS algorithm, phase amplitude couplings caused by steep modulation of the spectrum results in appearance of the group delay in the transient region. The group delay retrieved from the GS algorithm is larger and manifests itself even at large detuning from the transient region.

The calculated group delay was converted to pulse front duration with a positive stretching of $6 \mathrm{ps} / \mathrm{nm}$. The results in Fig. 8 were obtained for both DFS (c) and GS (d) algorithms. In either case, positive and negative RF chirp signs are analyzed. Reference curves show the pulse envelope without the effect of additional group delay. The front durations for the DFS algorithm are 3.5 and 2.9 ps; the front durations for the GS algorithm are 3.8 and $3.0 \mathrm{ps.}$ In both cases, positive $B_{2}$ results in larger rise/fall time, and this effect is stronger expressed for the GS algorithm. Those estimates correspond to experimental results.

We emphasize that the difference of modulation rise/fall time was observed only in time domain, while the steepness of modulation in spectral domain does not depend on $B_{2}$ sign both for DFS and GS algorithms (see Fig. 4 

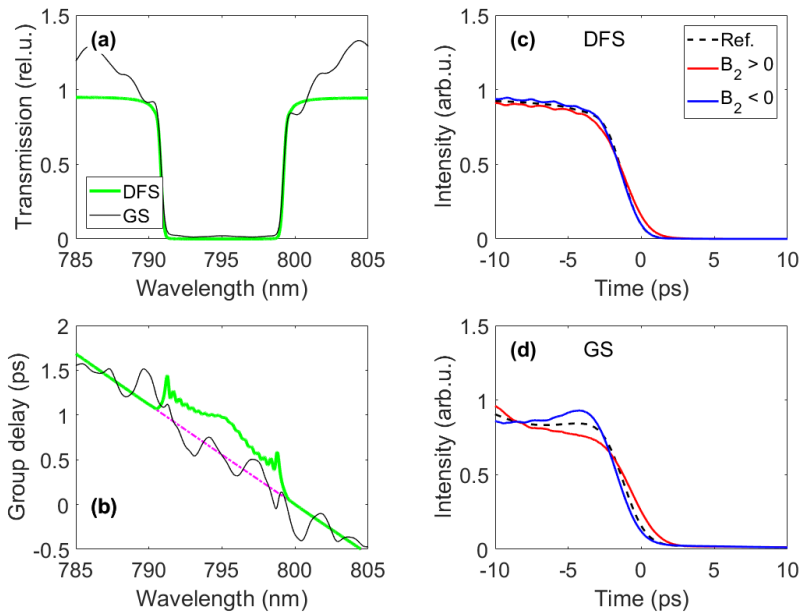

Figure 8: Effect of phase-amplitude couplings and finite AODDL transmission bandwidth: (a) transmission magnitude; (b) group delay at $B_{2}<0$, the case $B_{2}>0$ is similar with only the sign of the group delay changed; (c) pulse envelope for the DFS method; (d) pulse envelope for the GS algorithm.

(d)). This fact provides evidence in favor of the hypothesis that the group delay caused by non-quadratic spectral phase and phase-amplitude couplings affects modulation rise/fall time in chirped pulses.

\section{Conclusion}

Experimental study included binary spectral modulation, regenerative amplification, and direct time-domain measurement of chirped femtosecond laser pulses in Ti:sapphire emission band from 750 to $850 \mathrm{~nm}$. Average measured pulse fronts ranged from 3.6 to 6.7 ps depending on experimental settings. We discovered, that the sign of the SOD produced by the AODDL pulse shaper affects the rise/fall time of envelope modulation despite the absolute value of this SOD is on the order of $10^{-2}$ of the stretcher dispersion. The shortest pulse fronts of $3.6 \pm 0.6 \mathrm{ps}$ using explicit DFS method for RF waveform synthesis were obtained for the pulses with $6.2 \mathrm{ps} / \mathrm{nm}$ linear chirp when the AO-induced SOD was negative.

We observed that limited spectral resolution of the pulse shaper alters local spectral phase, if spectral modulation within the passband of the filter is large. Preset spectral modulation can be considered as binary because the software-defined amplitude step was several times narrower that the filter passband. Our experiments and related simulations showed that the effect is strong enough even with a high-resolution AODDL. Another effect that can contribute to the change of pulse front duration is optical path difference caused by spatial and angular chirps arising from anisotropy of quasi-collinear AO interaction.

Finally, we mention that the estimations of the dispersion and the group delay in Sec. 4 were obtained with phenomenological calculations. Rigorous theory of angu- lar chirp and phase-amplitude couplings in AODDLs requires analysis of $\mathrm{AO}$ ultrashort pulse diffraction in quasicollinear geometry considering the effects of acoustic beam divergence and anisotropy of phase matching. To our knowledge, such rigorous theory does not exist to date and will be a plot for future research.

\section{Acknowledgments}

The research was supported by the Ministry of Education and Science of the Russian Federation (075-02-20201588), National University of Science and Technology MISIS (Grant K2-2020-007), and the Russian Foundation for Basic Research (Project 18-07-00672).

[1] A. Rashidinejad, A.M. Weiner, Photonic radio-frequency arbitrary waveform generation with maximal time-bandwidth product capability, J. Lightwave Technol. 32 (20) (2014) 3383-3393. doi:10.1109/JLT.2014.2331491.

[2] Y. Mei, T. Jin, H. Chi, S. Zheng, X. Jin, X. Zhang, Characterization of the photonic generation of phase-coded RF signals based on pulse shaping and frequency-to-time mapping, Appl. Opt. 54 (13) (2015) 3956-3962. doi:10.1364/A0.54.003956

[3] K.B. Yushkov, V.V. Romanov, G.S. Rogozhnikov, V.Ya. Molchanov, $70 \mathrm{GHz}$ arbitrary modulation of chirped laser pulses by means of acousto-optics, Opt. Lett. 41 (23) (2016) 54425445. doi:10.1364/0L.41.005442

[4] C. Kerse, H. Kalaycioglu, P. Elahi, B. Cetin, D.K. Kesim, O Akcaalan, S. Yavas, M.D. Ask, B. Oktem, H. Hoogland, R. Holzwarth, F.O. Ilday, Ablation-cooled material removal with ultrafast bursts of pulses, Nature 537 (7618) (2016) 84-88. doi: 10.1038/nature18619

[5] F. Garrelie, F. Bourquard, A.-S. Loir, C.Donnet, J.-P. Colombier, Control of femtosecond pulsed laser ablation and deposition by temporal pulse shaping, Opt. Laser Technol. 78, Part A (2016) 42-51. doi:10.1016/j.optlastec.2015.09.020

[6] G. Bonamis, K. Mishchick, E. Audouard, C. Hönninger, E. Mottay, J. Lopez, I. Manek-Hönninger, High efficiency femtosecond laser ablation with gigahertz level bursts, J. Laser Appl. 31 (2) (2016) 022205. doi:10.2351/1.5096087.

[7] I. Astrauskas, B. Považay, A.Baltuška, A.Pugžlys, Influence of 2.09- $\mu \mathrm{m}$ pulse duration on through-silicon laser ablation of thin metal coatings, Opt. Laser Technol. 133 (2021) 106535. doi: $10.1016 / \mathrm{j}$. optlastec.2020.106535

[8] S. Yu. Guskov, Fast ignition of inertial confinement fusion targets, Plasma Physics Reports 39 (1) (2013) 1-50.

[9] S. Atzeni, A. Marocchino, A. Schiavi, G. Schurtz, Energy and wavelength scaling of shock-ignited inertial fusion targets, New J. Phys. 15 (4) (2013) 045004. doi:10.1088/1367-2630/15/4/ 045004

[10] R. Betti, O.A. Hurricane, Inertial-confinement fusion with lasers, Nat. Phys. 12 (5) (2016) 435-448. doi:10.1038/ NPHYS3736

[11] S.A. Belkov, S.V. Bondarenko, N.N. Demchenko, S.G. Garanin, S.Yu. Guskov, P.A. Kuchugov, V.B. Rozanov, R.V. Stepanov, R.A. Yakhin, N.V. Zmitrenko, Compression and burning of a direct-driven thermonuclear target under the conditions of inhomogeneous heating by a multi-beam megajoule laser, Plasma Phys. Control. Fusion 61 (2) (2019) 025011. doi:10.1088/ 1361-6587/aaf062

[12] D. Strickland, G. Mourou, Compression of amplified chirped optical pulses, Opt. Commun. 56 (3) (1985) 219-221. doi:10. 1016/0030-4018(85) 90120-8

[13] D.T. Reid, C.M. Heyl, R.R. Thomson, R. Trebino, G. Steinmeyer, H.H. Fielding, R. Holzwarth, Z. Zhang, P. DelHaye, T. Südmeyer, G. Mourou, T. Tajima, D. Faccio, F.J.M. Harren, G. Cerullo, Roadmap on ultrafast optics, J. Opt. 18 (9) (2016) 093006. doi:10.1088/2040-8978/18/9/093006 
[14] C.P.J. Barty, The Nexawatt: A strategy for exawatt peak power lasers based on NIF and NIF-like beam lines, J. Phys. Conf. Ser. 717 (2016) 012086.

[15] K. Ohno, T. Tanabe, F. Kannari, Adaptive pulse shaping of phase and amplitude of an amplified femtosecond pulse laser by direct reference to frequency-resolved optical gating traces, J. Opt. Soc. Am. B - Opt. Phys. 19 (11) (2002) 2781-2790.

[16] A. Rundquist, A. Efimov, D.H. Reitze, Pulse shaping with the Gerchberg-Saxton algorithm, J. Opt. Soc. Am. B - Opt. Phys. 19 (10) (2002) 2468-2478. doi:10.1364/JOSAB.19.002468

[17] V.Ya. Molchanov, S.I. Chizhikov, O.Yu. Makarov, N.P. Solodovnikov, V.N. Ginzburg, E.V. Katin, E.A. Khazanov, V.V. Lozhkarev, I.V. Yakovlev, Adaptive acousto-optic technique for femtosecond laser pulse shaping, Appl. Opt. 48 (7) (2009) C118C124. doi:10.1364/A0.48.00C118.

[18] M.Ya. Shchelev, Pico-femto-attosecond photoelectronics: looking through the lens of half a century, Phys. Usp. 55 (6) (2012) 607-614. doi:10.3367/UFNe.0182.201206f.0649

[19] N.S. Vorobiev, P.B. Gornostaev, V.I. Lozovoi, A.V. Smirnov, E.V. Shashkov, M.Ya. Schelev, A PS-1/S1 picosecond streak camera in experimental physics, Instrum. Exp. Tech. 59 (4) (2011) 551-556. doi:10.1134/S0020441216030246

[20] V.Ya. Molchanov, S.I. Chizhikov, K.B. Yushkov, Device for measuring transient characteristics of optical amplifiers, Patent RU 2650854 C1 of 17.04.2018.

[21] K.B. Yushkov, Acousto-optic programmable filters and the sampling theorem, Proc. SPIE 11210 (2019) 1121008. doi:10.1117/ 12.2539309

[22] N.V. Didenko, A.V. Konyashchenko, P.V. Kostryukov, L.L. Losev, V.S. Pazyuk, S.Yu. Tenyakov, V.Ya. Molchanov, S.I. Chizhikov, K.B. Yushkov, 40-fs hydrogen Raman laser, Quantum Electron. 45 (12) (2015) 1101-1104. doi:10.1070/ QE2015v045n12ABEH015906

[23] K.B. Yushkov, V.Ya. Molchanov, E.A. Khazanov, Uncertainty relation in broadband laser pulse shaping, Phys. Usp. 63 (2020) [in press]. doi:10.3367/UFNe.2020.06.038793.

[24] V.Ya. Molchanov, K.B. Yushkov, Advanced spectral processing of broadband light using acousto-optic devices with arbitrary transmission functions, Opt. Express 22 (13) (2014) 1566815678. doi:10.1364/OE.22.015668.

[25] K.B. Yushkov, V.Ya. Molchanov, A.V. Ovchinnikov, O.V. Chefonov, Acousto-optic replication of ultrashort laser pulses, Phys. Rev. A 96 (4) (2017) 043866. doi:10.1103/PhysRevA.96. 043866

[26] K.B. Yushkov, V.Ya. Molchanov, Randomly spaced phase-only transmission combs for femtosecond pulse shaping, IEEE J. Sel. Top. Quantum Electron. 26 (5) (2020) 8700108. doi:10.1109/ JSTQE. 2020.2984570.

[27] D.P. Kelly, Numerical calculation of the Fresnel transform, J. Opt. Soc. Am. A - Opt. Image Sci. Vis. 31 (4) (2014) 755-764. doi:10.1364/JOSAA.31.000755

[28] A. Weiner, Ultrafast Optics, Wiley, New York, 2009.

[29] O.E. Martinez, 3000 Times grating compressor with positive group velocity dispersion: Application to fiber compensation in 1.3-1.6 $\mu \mathrm{m}$ region, IEEE J. Quantum Electron. 23 (1) (1987) 59-64. doi:10.1109/JQE.1987.1073201.

[30] S̆. Vyhlídka, D. Kramer, G. Kalinchenko, B. Rus, Seidel aberrations in grating pulse stretchers, Opt. Express 24 (26) (2016) 30421-30432. doi:10.1364/0E.24.030421

[31] D.J. McCabe, D.R. Austin, A. Tajalli, S. Weber, I.A. Walmsley, B. Chatel, Spacetime coupling of shaped ultrafast ultraviolet pulses from an acousto-optic programmable dispersive filter, J. Opt. Soc. Am. B - Opt. Phys. 28 (1) (2011) 58-64. doi:10. 1364/JOSAB. 28.000058

[32] K. Osvay, M. Mero, Á. Börzsönyi, A.P. Kovács, M.P. Kalashnikov, Spectral phase shift and residual angular dispersion of an acousto-optic programmable dispersive filter, Appl. Phys. B - Lasers Opt. 107 (11) (2012) 125-130. doi:10.1007/ s00340-011-4867-7

[33] V.P. Zarubin, K.B. Yushkov, A.I. Chizhikov, O.Yu. Makarov, V.Ya. Molchanov, S.A. Tretiakov, A.I. Kolesnikov, E.B.
Cherepetskaya, A.A. Karabutov, Laser-ultrasonic temperature mapping of an acousto-optic dispersive delay line, NDT \& E. Int. 98 (2018) 171-176. doi:10.1016/j.ndteint.2018.05.010.

[34] S.P. Anikin, V.F. Esipov, V.Ya. Molchanov, A.M. Tatarnikov, K.B. Yushkov, An acousto-optical imaging spectrometer for astrophysical measurements, Opt. Spectrosc. 121 (1) (2016) 115122. doi:10.1134/S0030400X1607002X. 